\title{
Charm CPV and rare decays at LHCb
}

\author{
Michael J. Morello*i \\ Scuola Normale Superiore and INFN Sezione di Pisa \\ E-mail: michael.morellodsns.it
}

Charm physics has been playing all along a role in particle physics, by contributing to the formulation of the Standard Model (SM) as it is known nowadays. The level of attention on it has tremendously increased in recent years because of the first experimental observations of the slow mixing rate of the $D^{0}-\bar{D}^{0}$ flavour oscillations, providing definitely a full range of probes, entirely complementary to the $B$ and $K$ mesons, for mixing and $C P$ violation. In fact the charm quark is the only up-type quark that manifests flavour oscillations. Only in recent years it has been possible to collect huge and very clean samples of $D$ meson decays, several orders of magnitude larger in size than in the past, allowing also for the first time approaching the small SM expectations for $C P$-violation below the $10^{-3}$ level. Thus, the dynamics of the charm quark can be probed for the presence of New Physics with negligible SM "background", since any generic non-SM contribution would naturally carry additional $C P$-violating phases, which could enhance the observable $C P$-violation relative to $\mathrm{SM}$ predictions. It is worth to mention that $D^{0}-\bar{D}^{0}$ mixing can proceed in the SM through a double weak boson exchange (short distance contributions) or through intermediate states that are accessible to both $D^{0}$ and $\bar{D}^{0}$ (long distance contributions). The potentially large long distance contributions are non-perturbative and therefore difficult to estimate from the theory, however the interplay of many and even more precise experimental measurements, that only recently approached the desired precision, along with the foreseen theoretical improvements in calculating such amplitudes, should allow constraining theoretical uncertainties in the next and far future increasing the sensitivity to possible contributions from new processes and particles at mass scales beyond the reach of direct searches.

The LHCb experiment at the Large Hadron Collider is currently the main player and this brief write-up covers the most recent $\mathrm{LHCb}$ results on $\mathrm{CP}$-violation in the charm sector and on the search of rare charm decays.

16th International Conference on B-Physics at Frontier Machines

2-6 May 2016

Marseille, France

\footnotetext{
* Speaker.

'On behalf of the LHCb Collaboration.
} 


\section{Charm physics at the LHCb experiment: the beginning of a new era}

The main advantage of investigating charm physics in an hadronic environment, like the Large Hadron Collider (LHC), is the large production cross section of charm hadrons. An unprecedented huge amount of decays of all charm species was produced in Run 1 from $p p$ collisions at the LHC, with $\approx 5 \times 10^{12} D^{0}$ decays within the LHCb [1] acceptance corresponding to a cross section of $\approx 1600 \mu \mathrm{b}$ [2]. However the large $c \bar{c}$-cross-section has to be compared with the total inelastic $p p$ cross-section which is about 100 times larger. This means that despite their huge rate charm events are overwhelmed by an huge amount of background events. The task of improving such an unfavorable ratio between $c \bar{c}$ events and background is performed by the LHCb data acquisition and trigger systems that allow collecting high-purity and abundant samples of $D$ meson decays. In Run 1 the LHCb experiment collected $3 \mathrm{fb}^{-1}$ of integrated luminosity at an instantaneous luminosity of $4 \times 10^{32} \mathrm{~cm}^{-2} \mathrm{~s}^{-1}, 1 \mathrm{fb}^{-1}\left(2 \mathrm{fb}^{-1}\right)$ at $7 \mathrm{TeV}(8 \mathrm{TeV})$ center-of-mass energy. This luminosity corresponds to about $0.7 \times 10^{9}$ Cabibbo-favored untagged $D^{0} \rightarrow K^{-} \pi^{+}$decays, that become about $0.1 \times 10^{9}$ decays when the flavour of the $D^{0}$ meson is inferred by the charge of the soft pion from the strong $D^{*+} \rightarrow D^{0} \pi^{+}$decay. This leads to a 30 times larger sample than the full data sample collected by the precedent CDF II experiment. LHCb is currently taking data, the so called Run 2 , and it is expected to integrate until the end of $2018 \approx 6 \mathrm{fb}^{-1}$ of additional data. Moreover LHCb will resume data-taking in Run 3 (2021) with an upgraded detector at an higher instantaneous luminosity of $2 \times 10^{33} \mathrm{~cm}^{-2} \mathrm{~s}^{-1}$ aiming at integrating $50 \mathrm{fb}^{-1}$ of data until 2028 in 4 years. Considering that in Run 1 the total efficiency of the LHCb detector to collect charm decays is below $1 \%$, a large improvement in collecting an even larger sample of charm decays, well beyond the gain in integrated lunimosity, is conceivable. In this panorama current $\mathrm{LHCb}$ measurements represent the beginning of new era aiming at an extreme precise experimental exploration of the charm sector.

\section{Cabibbo-suppressed $D^{0} \rightarrow \pi^{+} \pi^{-}$and $D^{0} \rightarrow K^{+} K^{-}$decays}

Examples of clean experimental channels allowing the study of both direct and indirect $C P$ violation in the charm system are the neutral singly-Cabibbo-suppressed decays into $C P$-eigenstates, such as $D^{0} \rightarrow f$, with $f=K^{-} K^{+}$and $f=\pi^{-} \pi^{+}$. The time-dependent $C P$ asymmetry, $A_{C P}(f ; t)$, can be approximated by [3]

$$
A_{C P}(f ; t) \equiv \frac{\Gamma\left(D^{0}(t) \rightarrow f\right)-\Gamma\left(\bar{D}^{0}(t) \rightarrow f\right)}{\Gamma\left(D^{0}(t) \rightarrow f\right)+\Gamma\left(\bar{D}^{0}(t) \rightarrow f\right)} \approx a_{C P}^{\mathrm{dir}}(f)\left(1+\frac{t}{\tau} y_{C P}\right)+\frac{t}{\tau} a_{C P}^{\mathrm{ind}}, \quad(x, y \ll \tau / t)
$$

in terms of a direct component associated with $C P$ violation in the decay amplitudes $a_{C P}^{\mathrm{dir}}(f)$, and an indirect component associated with $C P$ violation in the mixing or in the interference between mixing and decay $a_{C P}^{\text {ind }}$. $\Gamma$ denotes the decay rate, $\tau$ the $D^{0}$ lifetime, $(x, y)$ are the $D^{0}$ mixing parameters, and $y_{C P}$ is the deviation from unity of the ratio of the effective lifetimes of decays to flavour specific and $C P$-even final states. In the limit of exact symmetry under a transformation interchanging $d$ and $s$ quarks (U-spin symmetry), the direct component is expected to be equal in magnitude and opposite in sign for $K^{-} K^{+}$and $\pi^{-} \pi^{+}$decays, however, large U-spin breaking effects could be present (see Ref. [4] and the references therein). The measured time-integrated asymmetry, $A_{C P}(f)$, 
depends upon the effective decay time acceptance. It can be written as [3]

$$
A_{C P}(f) \approx a_{C P}^{\mathrm{dir}}(f)\left(1+\frac{\langle t(f)\rangle}{\tau} y_{C P}\right)+\frac{\langle t(f)\rangle}{\tau} a_{C P}^{\mathrm{ind}},
$$

where $\langle t(f)\rangle$ denotes the mean decay time of reconstructed $D^{0} \rightarrow f$ decays. To a good approximation, $a_{C P}^{\text {ind }}$ is independent of the decay mode. Neglecting terms of the order $\mathscr{O}\left(10^{-6}\right)$, the difference in $C P$ asymmetries between $D^{0} \rightarrow K^{-} K^{+}$and $D^{0} \rightarrow \pi^{-} \pi^{+}$is

$$
\Delta A_{C P} \equiv A_{C P}\left(K^{-} K^{+}\right)-A_{C P}\left(\pi^{-} \pi^{+}\right) \approx \Delta a_{C P}^{\mathrm{dir}}\left(1+\frac{\overline{\langle t\rangle}}{\tau} y_{C P}\right)+\frac{\Delta\langle t\rangle}{\tau} a_{C P}^{\mathrm{ind}},
$$

where $\overline{\langle t\rangle}$ is the arithmetic average of $\left\langle t\left(K^{-} K^{+}\right)\right\rangle$and $\left\langle t\left(\pi^{-} \pi^{+}\right)\right\rangle$. From an experimental point of view the observed raw asymmetry, $A_{\text {raw }}(f)$, measured for $D^{0}$ decays to a final state $f$ is defined as

$$
A_{\text {raw }}(f) \equiv \frac{N\left(D^{*+} \rightarrow D^{0}(f) \pi_{s}^{+}\right)-N\left(D^{*-} \rightarrow \bar{D}^{0}(f) \pi_{s}^{-}\right)}{N\left(D^{*+} \rightarrow D^{0}(f) \pi_{s}^{+}\right)+N\left(D^{*-} \rightarrow \bar{D}^{0}(f) \pi_{s}^{-}\right)},
$$

where $N$ is the number of reconstructed signal candidates of the given decay and the flavour of the $D^{0}$ meson is identified using the charge of the soft pion $\left(\pi_{s}^{+}\right)$in the strong decay $D^{*+} \rightarrow D^{0} \pi_{s}^{+}$ ( $D^{*}$-tag). The raw asymmetry can be written as

$$
A_{\text {raw }}(f) \approx A_{C P}(f)+A_{\mathrm{D}}(f)+A_{\mathrm{D}}\left(\pi_{s}^{+}\right)+A_{\mathrm{P}}\left(D^{*+}\right),
$$

where $A_{\mathrm{D}}(f)$ and $A_{\mathrm{D}}\left(\pi_{s}^{+}\right)$are the asymmetries of the detection efficiencies of the $D^{0}$ final state and of the soft pion, and $A_{\mathrm{P}}\left(D^{*+}\right)$ is the production asymmetry for $D^{*+}$ mesons, arising from the hadronisation of charm quarks in $p p$ collisions. The magnitudes of $A_{\mathrm{P}}\left(D^{*+}\right)$ and $A_{\mathrm{D}}\left(\pi_{s}^{+}\right)$are both about $1 \%$. Equation (2.4) is only valid when the detection efficiencies of the final state $f$ and of the soft pion are independent. Since both $K^{-} K^{+}$and $\pi^{-} \pi^{+}$final states are self-conjugate, $A_{\mathrm{D}}\left(K^{-} K^{+}\right)$and $A_{\mathrm{D}}\left(\pi^{-} \pi^{+}\right)$are identically zero. To a good approximation $A_{\mathrm{D}}\left(\pi_{s}^{+}\right)$and $A_{\mathrm{P}}\left(D^{*+}\right)$ are independent of the final state $f$ in any given kinematic region, and thus cancel in the difference $\Delta A_{C P}=A_{\text {raw }}\left(K^{-} K^{+}\right)-A_{\text {raw }}\left(\pi^{-} \pi^{+}\right)$. However, to take into account an imperfect cancellation of detection and production asymmetries due to the difference in the kinematic properties of the two decay modes, the kinematic distributions of $D^{*+}$ mesons decaying to the $K^{-} K^{+}$final state are reweighted to match those of $D^{*+}$ mesons decaying to the $\pi^{-} \pi^{+}$final state. The time-integrated difference between the $C P$ asymmetries in $D^{0} \rightarrow K^{-} K^{+}$and $D^{0} \rightarrow \pi^{-} \pi^{+}$decays is measured to be $\Delta A_{C P}=(-0.10 \pm 0.08$ (stat) \pm 0.03 (syst) $) \%[4]$ and this is the most precise measurement of a time-integrated $C P$ asymmetry in the charm sector from a single experiment. This result is compatible with the previous $\mathrm{LHCb}$ result [5] and with the same measurement performed with $D$-mesons from semileptonic $B \rightarrow D^{0} \mu^{-} v_{\mu} X$ decays (semileptonic-tag), where the $D^{0}$ flavour is inferred from the muon charge, $\Delta A_{C P}=(-0.14 \pm 0.16$ (stat) \pm 0.08 (syst) $) \%[6]$.

The time dependent term of Eq. (2.1) is related to the $A_{\Gamma}$ observable, defined as

$$
A_{\Gamma} \equiv \frac{\hat{\Gamma}\left(D^{0} \rightarrow f\right)-\hat{\Gamma}\left(\bar{D}^{0} \rightarrow f\right)}{\hat{\Gamma}\left(D^{0} \rightarrow f\right)+\hat{\Gamma}\left(\bar{D}^{0} \rightarrow f\right)}
$$

where $\hat{\Gamma}$ is the effective decay width defined as $1 / \hat{\Gamma}=\int t \Gamma(t) d t / \int \Gamma(t) d t$, where $\Gamma(t)$ is the time dependent decay rate. By neglecting in Eq. (2.1) the term $a_{C P}^{\mathrm{dir}}(f) y_{C P}$ with respect to $a_{C P}^{\text {ind }}$ one obtains 
$A_{\Gamma} \approx-a_{C P}^{\text {ind }}$ and that is why it is considered as a measurement of the indirect component of the $C P$ asymmetry. LHCb performed $A_{\Gamma}$ measurements both with the $D^{*}$-tag and with the semileptonictag, to infer the flavour of the $D^{0}$ at production. The most precise measurement is that with the $D^{*}$ tag on $1 \mathrm{fb}^{-1}$ of data, resulting $A_{\Gamma}\left(D^{0} \rightarrow K^{+} K^{-}\right)=(-0.35 \pm 0.62$ (stat) \pm 0.12 (syst) $) \times 10^{-3}$ [7] and $A_{\Gamma}\left(D^{0} \rightarrow \pi^{+} \pi^{-}\right)=(+0.33 \pm 1.06$ (stat) \pm 0.14 (syst) $) \times 10^{-3}$ [7]. Similar results are obtained with the semileptonic tag on $3 \mathrm{fb}^{-1}$ with a slightly worse precision as reported in Ref. [8].

Assuming that indirect $C P$ violation is independent of the $D^{0}$ final state, and combining the $\Delta A_{C P}$ measurements with the measurements of indirect $C P$ violation the values of the direct and indirect $C P$ asymmetry are found to be $a_{C P}^{\text {ind }}=(0.058 \pm 0.044) \%$ and $\Delta a_{C P}^{\mathrm{dir}}=(-0.061 \pm 0.076) \%$. Results summarized in the $\left(\Delta a_{C P}^{\mathrm{dir}}, a_{C P}^{\text {ind }}\right)$ plane are shown in Fig. 1 . They are consistent with the hypothesis of no- $C P$ asymmetry with a $p$-value of 0.32 [4].

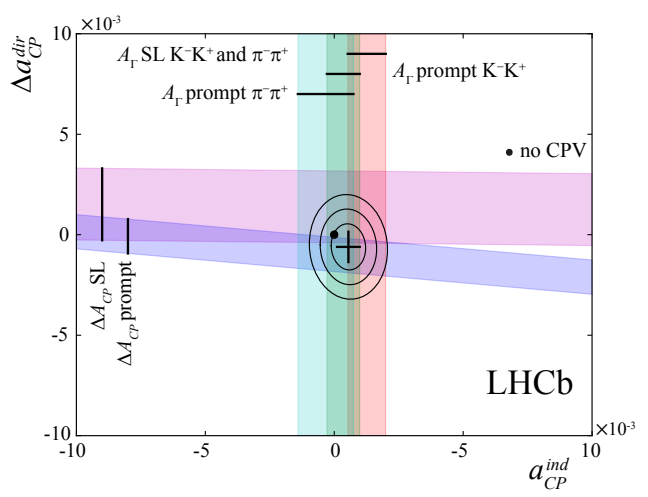

Figure 1: Contour plot of $\Delta a_{C P}^{\mathrm{dir}}$ versus $a_{C P}^{\text {ind }}$. The point at $(0,0)$ denotes the hypothesis of no $C P$ violation. The solid bands represent the measurements in Refs. [4, 6, 7, 8]. The value of $y_{C P}$ is taken from Ref. [9]. The contour lines shows the $68 \%, 95 \%$ and $99 \%$ confidence-level intervals from the combination.

\section{Observation of $D^{0}-\bar{D}^{0}$ mixing in multi-body decays}

LHCb recently reported on the measurement of the $D^{0}$-mixing by measuring the time-dependent ratio of $D^{0} \rightarrow K^{+} \pi^{-} \pi^{+} \pi^{-}$to $D^{0} \rightarrow K^{-} \pi^{+} \pi^{-} \pi^{+}$decay rates, where the flavour of the $D$ meson at production is determined using the $D^{*}$-tag [10]. The wrong-sign (WS) decay $D^{0} \rightarrow K^{+} \pi^{-} \pi^{+} \pi^{-}$ has two dominant contributions: a doubly Cabibbo-suppressed (DCS) amplitude, and a $D^{0}-\bar{D}^{0}$ oscillation followed by a Cabibbo-favoured (CF) amplitude. The right-sign (RS) decay $D^{0} \rightarrow$ $K^{-} \pi^{+} \pi^{-} \pi^{+}$is dominated by the CF amplitude, and has negligible contribution of $\mathscr{O}\left(10^{-4}\right)$ from $D^{0}-\bar{D}^{0}$ oscillations. Ignoring $C P$ violation, to second order in $t / \tau$, the time-dependence of the phase-space integrated decay rate ratio $R(t)$ is approximated by

$$
R(t)=\frac{\Gamma\left[D^{0} \rightarrow K^{+} \pi^{-} \pi^{+} \pi^{-}\right](t)}{\Gamma\left[D^{0} \rightarrow K^{-} \pi^{+} \pi^{-} \pi^{+}\right](t)} \approx\left(r_{D}^{K 3 \pi}\right)^{2}-r_{D}^{K 3 \pi} R_{D}^{K 3 \pi} \cdot y_{K 3 \pi}^{\prime} \frac{t}{\tau}+\frac{x^{2}+y^{2}}{4}\left(\frac{t}{\tau}\right)^{2},
$$

where $\Gamma$ denotes the decay rate, $t$ is the measured proper decay-time of the $D^{0}$ meson, $\tau$ is the $D^{0}$ lifetime, and $r_{D}^{K 3 \pi}$ gives the phase space averaged ratio of the DCS to the CF amplitudes. Here, $y_{K 3 \pi}^{\prime}$ is defined by $y_{K 3 \pi}^{\prime} \equiv y \cos \delta_{D}^{K 3 \pi}-x \sin \delta_{D}^{K 3 \pi}$, where $\delta_{D}^{K 3 \pi}$ is the average strong phase difference; 
this and the coherence factor, $R_{D}^{K 3 \pi}$, are defined by $R_{D}^{K 3 \pi} e^{-i \delta_{D}^{K 3 \pi}} \equiv\langle\cos \delta\rangle+i\langle\sin \delta\rangle$, where $\langle\cos \delta\rangle$ and $\langle\sin \delta\rangle$ are the cosine and sine of the phase of the ratio of the DCS to the CF amplitude, averaged over phase space. All three parameters, $r_{D}^{K 3 \pi}, R_{D}^{K 3 \pi}$ and $\delta_{D}^{K 3 \pi}$ are required to determine the CKM angle $\gamma$ in $B^{+} \rightarrow D K^{+}, D \rightarrow K^{-} \pi^{+} \pi^{-} \pi^{+}$decays. Using the full Run 1 data sample LHCb is sensitive to the phase-space averaged ratio of DCS to CF amplitudes $r_{D}^{K 3 \pi}$ and the product of the coherence factor $R_{D}^{K 3 \pi}$ and a charm mixing parameter $y_{K 3 \pi}^{\prime}$. The constraints measured are $r_{D}^{K 3 \pi}=(5.67 \pm 0.12) \times 10^{-2}$ [10], which is the most precise determination to date, and $R_{D}^{K 3 \pi}$. $y_{K 3 \pi}^{\prime}=(0.3 \pm 1.8) \times 10^{-3}$ [10]. The analysis also gives the most precise measurement of the $D^{0} \rightarrow K^{+} \pi^{-} \pi^{+} \pi^{-}$branching fraction, and the first observation of $D^{0}-\bar{D}^{0}$ oscillations in this decay mode, with a significance of $8.2 \sigma$.

\section{Rare charm decays}

Thanks to the excellent capabilities in rejecting backgrounds keeping high signal efficiencies, LHCb is also a special place to search for rare decays of charm mesons, such as flavour changing neutral current processes (FCNC) and the lepton flavour violating processes. The former are highly suppressed within the SM, while the latter are forbidden at all. These decays therefore probe potential contributions from new processes and particles at mass scales beyond the reach of direct searches. LHCb recently reported the first observation of the $D^{0} \rightarrow K^{-} \pi^{+} \mu^{+} \mu^{-}$ decay using $2 \mathrm{fb}^{-1}$ of data collected in 2012 at a centre-of-mass energy of $8 \mathrm{TeV}$, being that a reference for measurements of FCNC $c \rightarrow u \mu^{+} \mu^{-}$transitions, such as $D^{0} \rightarrow \pi^{+} \pi^{-} \mu^{+} \mu^{-}$and $D^{0} \rightarrow K^{+} K^{-} \mu^{+} \mu^{-}$. The branching fraction in the dimuon mass range $675-875 \mathrm{MeV} / c^{2}$ is measured to be $\mathscr{B}\left(D^{0} \rightarrow K^{-} \pi^{+} \mu^{+} \mu^{-}\right)=(4.17 \pm 0.12$ (stat) \pm 0.40 (syst) $) \times 10^{-6}$ [11], and can be compared to the SM value calculated in Ref. [12], $6.7 \times 10^{-6}$, in the full dimuon mass range. The branching fraction is measured with an overall precision of $10 \%$ and is one order of magnitude lower than the previous most stringent upper limit. LHCb has also recently searched for the $D^{0} \rightarrow e^{ \pm} \mu^{\mp}$ decay [13], which is an example of a forbidden decay, in which lepton flavour is not conserved. The contributions to this process from neutrino oscillations would result into a rate that is well below the reach of any currently feasible experiment. However, the decay is predicted to occur in several other models that extend the SM, with rates varying by up to eight orders of magnitude. LHCb analysed the full Run 1 dataset. Candidate $D^{0}$ mesons are selected using the decay $D^{*+} \rightarrow D^{0} \pi^{+}$and the possible $D^{0} \rightarrow e^{ \pm} \mu^{\mp}$ production is studied using the decay mode $D^{0} \rightarrow K^{-} \pi^{+}$as a normalisation channel. No significant excess of $D^{0} \rightarrow e^{ \pm} \mu^{\mp}$ candidates over the expected background is seen, and a limit is set on the branching fraction, $\mathscr{B}\left(D^{0} \rightarrow e^{ \pm} \mu^{\mp}\right)<1.3 \times 10^{-8}$, at $90 \%$ confidence level [13]. This is an order of magnitude lower than the previous limit and it further constrains the parameter space in some leptoquark models and in supersymmetric models with R-parity violation [14].

\section{Conclusions}

LHC is the most copious source of charm decays ever and LHCb collected the largest data samples of charm decays. In Run 1 statistical precisions below $\mathscr{O}\left(10^{-3}\right)$ have been already achieved in the measurements of $C P$ violating asymmetries (especially for two-body golden modes), with 
systematic uncertainties already close to the interesting level of $\mathscr{O}\left(10^{-4}\right)$, approaching for the first time the upper bounds of SM expectations. No hints of $C P$-violation or anomalies have been found so far. Limits on rare charm decays have been improved by several orders of magnitude with respect to the past.

$\mathrm{LHCb}$ is currently taking data and it is conceivable that the size of the $D$ meson data sample will increase more than proportionally to the integrated luminosity, both in Run 2 and after in the LHCb-Upgrade, opening a privileged door for studying at very high precision the structure of flavour dynamics, and consequently effects of New Physics. The exploration of the charm sector at an unprecedented level of precision nowadays is extremely important not only by itself, as a probe for the presence of new phenomena, but also because it proves that future measurements on beauty sector, with a precision similar (or even better) to the one currently available in the charm sector, will be feasible and the systematic uncertainties will be under control. This is certainly true for the upgraded LHCb-detector, and likely it will continue to be true for possible future LHC flavour experiments at much higher intensity.

\section{References}

[1] A. A. Alves, Jr. et al. [LHCb Collaboration], JINST 3, S08005 (2008); R. Aaij et al. [LHCb Collaboration], Int. J. Mod. Phys. A 30, no. 07, 1530022 (2015); [arXiv:1412.6352 [hep-ex]].

[2] R. Aaij et al. [LHCb Collaboration], Nucl. Phys. B 871, 1 (2013), [arXiv:1302.2864 [hep-ex]].

[3] T. Aaltonen et al. [CDF Collaboration], Phys. Rev. D 85, 012009 (2012), [arXiv:1111.5023 [hep-ex]]; M. Gersabeck et al., J. Phys. G 39, 045005 (2012), [arXiv:1111.6515 [hep-ex]].

[4] R. Aaij et al. [LHCb Collaboration], Phys. Rev. Lett. 116, no. 19, 191601 (2016), [arXiv:1602.03160 [hep-ex]].

[5] R. Aaij et al. [LHCb Collaboration], Phys. Rev. Lett. 108, 111602 (2012), [arXiv:1112.0938 [hep-ex]].

[6] R. Aaij et al. [LHCb Collaboration], JHEP 1407, 041 (2014), [arXiv:1405.2797 [hep-ex]].

[7] R. Aaij et al. [LHCb Collaboration], Phys. Rev. Lett. 112, no. 4, 041801 (2014), [arXiv:1310.7201 [hep-ex]].

[8] R. Aaij et al. [LHCb Collaboration], JHEP 1504, 043 (2015), [arXiv:1501.06777 [hep-ex]].

[9] R. Aaij et al. [LHCb Collaboration], JHEP 1204, 129 (2012), [arXiv:1112.4698 [hep-ex]].

[10] R. Aaij et al. [LHCb Collaboration], Phys. Rev. Lett. 116, no. 24, 241801 (2016), [arXiv:1602.07224 [hep-ex]].

[11] R. Aaij et al. [LHCb Collaboration], Phys. Lett. B 757, 558 (2016), [arXiv:1510.08367 [hep-ex]].

[12] L. Cappiello, O. Cata and G. D' Ambrosio, JHEP 1304, 135 (2013), [arXiv:1209.4235 [hep-ph]].

[13] R. Aaij et al. [LHCb Collaboration], Phys. Lett. B 754, 167 (2016), [arXiv:1512.00322 [hep-ex]].

[14] G. Burdman et al., Phys. Rev. D 66, 014009 (2002), [hep-ph/0112235]; F. Tahir et al., Chin. Phys. C38 (2014) 123101; R.-M. Wang et al., Int. J. Mod. Phys. A29 (2014), no. 291450169. 